\title{
THE EFFECT OF DIFFERENTIAL LIMB MAGNIFICATION ON ABUNDANCE ANALYSIS OF MICROLENSED DWARF STARS
}

\author{
Jennifer A. Johnson, Subo Dong ${ }^{1}$, And Andrew Gould \\ Department of Astronomy, Ohio State University, 140 W. 18th Ave., Columbus, OH 43210, USA; jaj@ astronomy.ohio-state.edu, \\ dong@astronomy.ohio-state.edu, gould@ astronomy.ohio-state.edu \\ Received 2009 October 16; accepted 2010 February 10; published 2010 March 25
}

\begin{abstract}
Finite source effects can be important in observations of gravitational microlensing of stars. Near caustic crossings, for example, some parts of the source star will be more highly magnified than other parts. The spectrum of the star is then no longer the same as when it is unmagnified, and measurements of the atmospheric parameters and abundances will be affected. The accuracy of abundances measured from spectra taken during microlensing events has become important recently because of the use of highly magnified dwarf stars to probe abundance ratios and the abundance distribution in the Galactic bulge. In this paper, we investigate the importance of finite source effects on spectra by using magnification profiles motivated by two events to synthesize spectra for dwarfs between $5000 \mathrm{~K}$ and $6200 \mathrm{~K}$ at solar metallicity. We adopt the usual techniques for analyzing the microlensed dwarfs, namely, spectroscopic determination of temperature, gravity, and microturbulent velocity, relying on equivalent widths. We find that ignoring the finite source effects for the more extreme case results in errors in $T_{\text {eff }}<45 \mathrm{~K}$, in $\log g$ of $<0.1$ dex, and in $\xi$ of $<0.1 \mathrm{~km} \mathrm{~s}^{-1}$. In total, changes in equivalent widths lead to small changes in atmospheric parameters and changes in abundances of $<0.06 \mathrm{dex}$, with changes in $[\mathrm{Fe} \mathrm{I} / \mathrm{H}]$ of $<0.03 \mathrm{dex}$. For the case with a larger source-lens separation, the error in $[\mathrm{Fe} \mathrm{I} / \mathrm{H}]$ is $<0.01 \mathrm{dex}$. This latter case represents the maximum effect seen in events whose light curves are consistent with a point-source lens, which includes the majority of microlensed bulge dwarfs published so far.
\end{abstract}

Key words: Galaxy: bulge - gravitational lensing: micro - line: profiles - stars: abundances - stars: atmospheres

Online-only material: color figures

\section{INTRODUCTION}

A microlensing event occurs when a stellar mass object passes between the observer and a background source. For events in the Local Group, the source is a star. If the source is finite, rather than infinitely small, parts of the source may be magnified more than other parts. In the case of stars, for example, the limb can be brightened relative to an unmagnified source. Since the light from the limb comes from different temperature profiles in the star compared to the center, this will affect the spectrum (e.g., Valls-Gabaud 1995; Loeb \& Sasselov 1995).

Recently, observers using large telescopes have published abundance ratios in dwarfs in the Galactic bulge from spectra obtained while the sources were highly magnified. A number of these have turned out to have super-solar metallicities, including OGLE-2006-BLG-265S (Johnson et al. 2007), MOA-2006-0BLG-099S (Johnson et al. 2008), OGLE-2007BLG-349S (Cohen et al. 2008), MOA-2008-BLG-310S and MOA-2008-BLG-311S (Cohen et al. 2009), and OGLE-2007BLG-514S (Epstein et al. 2010). Some are metal-poor, including the subgiant OGLE-2008-BLG-209S (Bensby et al. 2009a) and the dwarf OGLE-2009-BLG-076S (Bensby et al. 2009b), which at $[\mathrm{Fe} / \mathrm{H}]=-0.76$ is the most metal-poor dwarf/subgiant observed when microlensed. For a sample of eight microlensed dwarfs, Epstein et al. (2010) found that a K-S test gave a 1.6\% chance that the dwarfs were drawn from the same metallicity distribution function (MDF) as the giant MDF from Zoccali et al. (2008). Bensby et al. (2010) present the most up-to-date results for 14 bulge dwarf spectra, and find a much larger chance (30\%) that they were drawn from the same metallicity distribution as the giants. Cohen et al. (2008) proposed that mass-loss on the

\footnotetext{
1 Current address: Institute for Advanced Study, 1 Einstein Drive, Princeton, NJ 08540, USA.
}

giant branch prevents some more metal-rich stars from becoming red giants, similar to the mechanism suggested by Kalirai et al. (2007) to explain the low-mass He white dwarfs in the metal-rich cluster NGC 6791. However, this explanation predicts that there is a drop in the RGB luminosity function which is not observed, at least in the field observed by Zoccali et al. (2003). Bensby et al. (2010) note that if only the more metalrich stars are subject to missing part of red giant branch evolution, the effect on the whole bulge may not be large. Another explanation is that there are systematic differences between giant and dwarf abundance analyses. Cohen et al. (2009) calculated that a systematic offset of 0.10 dex between the dwarf and giant metallicity scales would give the mean metallicity offset a significance of $2 \sigma$ and that larger systematic offsets would obviously decrease the significance even further. Finally, Zoccali et al. (2008) suggested that the spectra of microlensed dwarfs could be affected by differential magnification sufficiently that the usual analysis of the dwarfs, which does not take this into account, could lead to biased answers and help explain the possible discrepancy. This last suggestion can be tested by comparing the answers obtained from synthetic spectra with and without differential limb magnification (DLM).

While most microlensing events follow the light curve of a point source, about $\sim 3 \%$ (Witt 1995) of events show finite source effects. This fraction is even higher for highmagnification events that are targets of the current generation of dwarf studies. Of the first eight published events from the current group of studies for which dwarf spectra were obtained, we know that at least three of the events were affected by finite source effects, namely OGLE-2007-BLG-514, OGLE2007-BLG-349, and MOA-2008-BLG-310. We need to determine the size of the effect that DLM has on the spectra and the measurement of the effective temperature $\left(T_{\text {eff }}\right)$, gravity 
$(\log g)$, metallicity $([\mathrm{Fe} / \mathrm{H}])$, microturbulent velocity $(\xi)$, and abundance ratios to correctly interpret these events.

In addition to probing the chemical evolution of the bulge, the accuracy of the measured $T_{\text {eff }}$ from the spectrum is important for testing the method by which colors of source stars in microlensing events are determined. Using a metallicity and a $T_{\text {eff }}$ derived from the spectrum, we can predict the color of the star using relations between color and $T_{\text {eff }}$, such as that by Ramírez \& Meléndez (2005), and compare with the color estimated using standard microlensing techniques, which rely on the offset of the star from the red clump. The results so far indicate that if the color of the red clump is $(V-I)_{0}=1.05$, the $T_{\text {eff }} \mathrm{s}$ derived spectra are in agreement with $T_{\text {eff }} \mathrm{s}$ from colors.

Much work has been done on the theoretical effects of DLM of the disk of giants during a microlensing event because these events are easier to find and are longer-lasting than similar events in dwarfs. (e.g., Valls-Gabaud 1998; Heyrovský et al. 2000; Gaudi \& Gould 1999), although none of these address the quantitative effect on the abundances. Finite source effects were observed for the first time for the giant MACHO 95BLG-30 (Alcock et al. 1997), and, thanks to intensive monitoring by observers, the effect of the size of giants on microlensing events has been observed many times (e.g., Albrow et al. 1999, 2001; Castro et al. 2001; Afonso et al. 2001; Cassan et al. 2004, 2006; Kubas et al. 2005; Thurl et al. 2006).

Studies of dwarf stars have been much rarer. Afonso et al. (2000) and Abe et al. (2003) reported the only measurements of limb darkening in dwarfs for MACHO 98 SMC-1, a mainsequence A star, and MOA 2002-BLG-33S, a solar-type star, respectively. The most relevant work to this paper is the analysis of Lennon et al. (1996) of the event MACHO 96-BLG-3. They took three exposures when the source, a dwarf star, was moving across a caustic. These $R \sim 1100$ spectra with signalto-noise ratio $(\mathrm{S} / \mathrm{N})$ of $25-100$ did not show any convincing cases of profile variability. Lennon et al. (1996) compared the observations of this star with both a library of high-resolution, high $\mathrm{S} / \mathrm{N}$ spectra of $\mathrm{F}$ and $\mathrm{G}$ stars observed at Calar Alto and a grid of synthetic spectra, and derived stellar parameters of $T_{\text {eff }}=6100 \mathrm{~K}$ from the $\mathrm{H} \alpha$ line, $\log g=4.25$ from the $\mathrm{Mg}$ I triplet and a metallicity $([\mathrm{M} / \mathrm{H}])$ between 0.3 and 0.6 from fits to regions with many $\mathrm{Fe}_{\mathrm{I}}$ and $\mathrm{Ca}$ I lines. They calculated the expected deviations from the unmagnified spectrum for this event, and found that the expected change in the line profiles for the three spectra was $\leqslant 1 \%$, while the expected change in the continuum was $\leqslant 2 \%$. Given the small changes expected and the $\mathrm{S} / \mathrm{N}$ of the spectra, it is not surprising that no changes were observed, and that the derived atmospheric parameters of the star would also not be affected. Indeed, it is not surprising that most changes are small. The amount of magnification depends on the distance from the lens, but the emitted spectrum is the same for an entire annulus (for a spherical star). Because the distance from the lens varies around the annulus, the average magnification of a spectrum at a particular annulus is smaller than the largest magnification of a particular spot would suggest. In addition, while the spectrum of the star increasingly changes from center to limb, the intensity of the limb is lower than the center by factor of a few. Therefore, it is difficult to overcome the influence of the light coming from the central regions of the star in the disk-averaged light.

The work discussed above focused on learning about the atmosphere of the star and showed that finite source effects cause changes in the spectrum of the star, which can be inverted to give temperature as a function of depth. Because the same

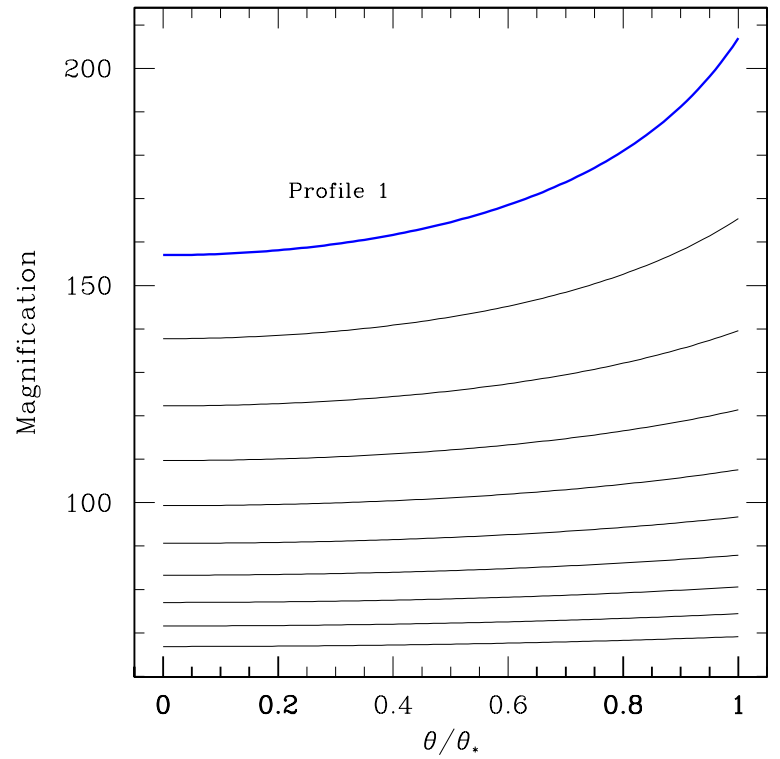

Figure 1. DLM profiles for Event A. The bold line shows Profile 1, which represents the most extreme differential magnification for this event, and is the profile from the first 15 minutes of Event A. At most, the limb is magnified by $\sim 30 \%$ more than the center.

(A color version of this figure is available in the online journal.)

effects that make these extreme events interesting as probes of the atmosphere will also change the spectrum that is analyzed for elemental abundances, the goal of this paper is to determine the effect of DLM on the $T_{\text {eff }}, \xi, \log g,[\mathrm{Fe} / \mathrm{H}]$, and abundance ratios using the standard spectroscopic techniques, such as equivalent width analysis. Therefore, we will be concerned with mimicking standard abundance analysis as closely as possible, rather than exploring the full range of knowledge that can be gleaned from time-resolved spectroscopy of microlensed events.

\section{MAGNIFICATION PROFILES DURING MICROLENSING EVENTS}

Our work was motivated by two events, MOA-2008BLG-310 and OGLE-2007-BLG-514, which are illustrations of the kind of effects seen. For MOA-2008-BLG-310, the lens did in fact transit the source, and there were small perturbations during this transit caused by a planetary companion in the lens (Janczak et al. 2010). However, the spectrum analyzed by Cohen et al. (2009) began at UT 22:51, which was 21 minutes after the end of the transit, when the lens and source center were separated by $z=1.22$ source radii. Figure 1 shows the annulus-averaged profiles for a point lens at 15 minute intervals for the MOA-2008-BLG-310 geometry, beginning at $z=1.2$ and continuing as the lens and source moved further apart. The magnification is given as a function of $\theta$, which is the angle between the normal to the stellar surface and the line of sight to the observer. This look at the later stages of MOA-2008BLG-310 will be referred to as Event A. The start time for the first profile is three minutes before the Cohen et al. (2009) observations began, and thus serve as a direct measurement of the size of the effect of DLM on those abundances, but they also serve a broader purpose.

In this particular case, we know that $z=1.22$ at the time of the observations because the source size was earlier detected by observations (from Africa) of a direct source crossing. However, if the closest separation between source and lens 


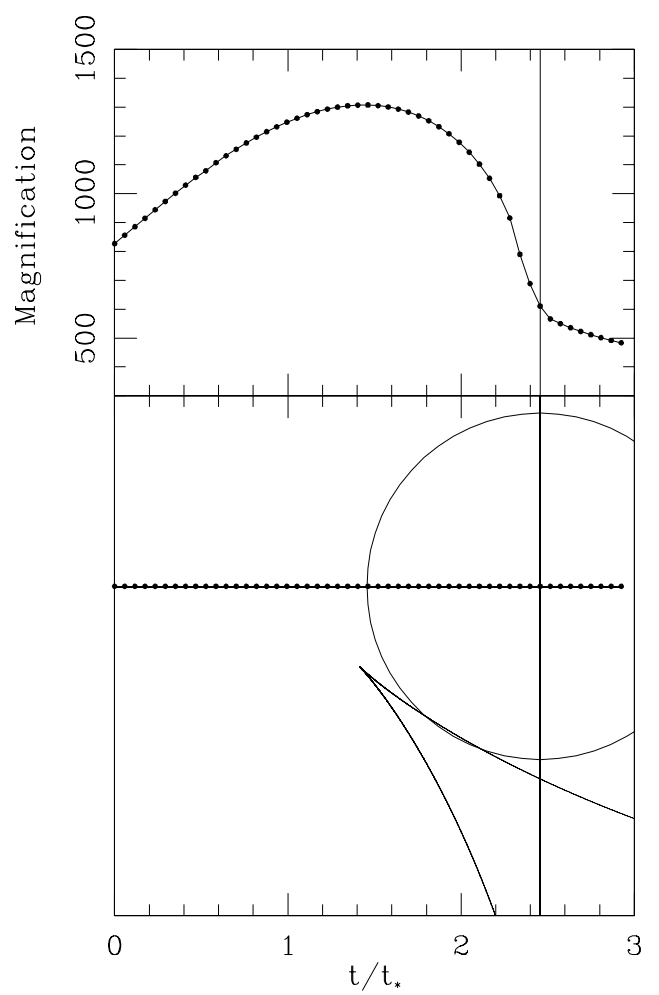

Figure 2. Top panel shows, for Event B, the source-averaged magnification as a function of time, measured in units of the source-radius crossing time. The bottom panel shows the geometry of the event at a moment when the source (circle) is just exiting the caustic (acute-angled structure), which is a contour of formally infinite magnification. The source-center position is marked by small circles at 5 minute intervals. As the source passes over the caustic, it is differentially magnified, with first the limb, then the center, and finally the limb being the most magnified.

during the entire event (i.e., the impact parameter) had been $z_{0}=1.22$, there would have been no source crossing at any time. If the impact parameter is sufficiently large, then it becomes impossible to determine the source radius from the light curve. In such a case, only upper limits can be placed on the magnitude of DLM and profiles of the DLM as a function of $\theta$ cannot be derived.

However, for $z_{0} \leqslant 1.2$, the effects of the finite-source size on the light curve are sufficiently pronounced to measure $z_{0}$. Thus, the top curve in Figure 1 represents the most extreme case of DLM that would occur without being noticed, and thus serves as a general check on ignoring DLM when it is not detectable from the light curve.

The other case represents a more extreme event, inspired by OGLE-2007-BLG-514, and will be called Event B. Here, the source trajectory crossed a cusp from a binary lens and produced extreme magnification variations (Figure 2). Once the parameters are selected, it is straightforward to compute the magnification at each time and for every point on the source plane. We calculated the mean magnification in concentric rings with annuli equal to 0.01 source radii using the "looplinking" technique (Dong et al. 2006). The profiles are spaced at 5 minute intervals for this event. Actual observations are always longer, usually 5-6 $\times$ longer in order to get enough $\mathrm{S} / \mathrm{N}$ in each exposure to reliably extract the spectrum. Spectra at 5 minute intervals could only be obtained for dwarfs magnified to apparent magnitudes that have not been seen in an event to date, so in reality microlensing spectra will smear out these profiles and dilute their effects. Figure 3 shows 51 different

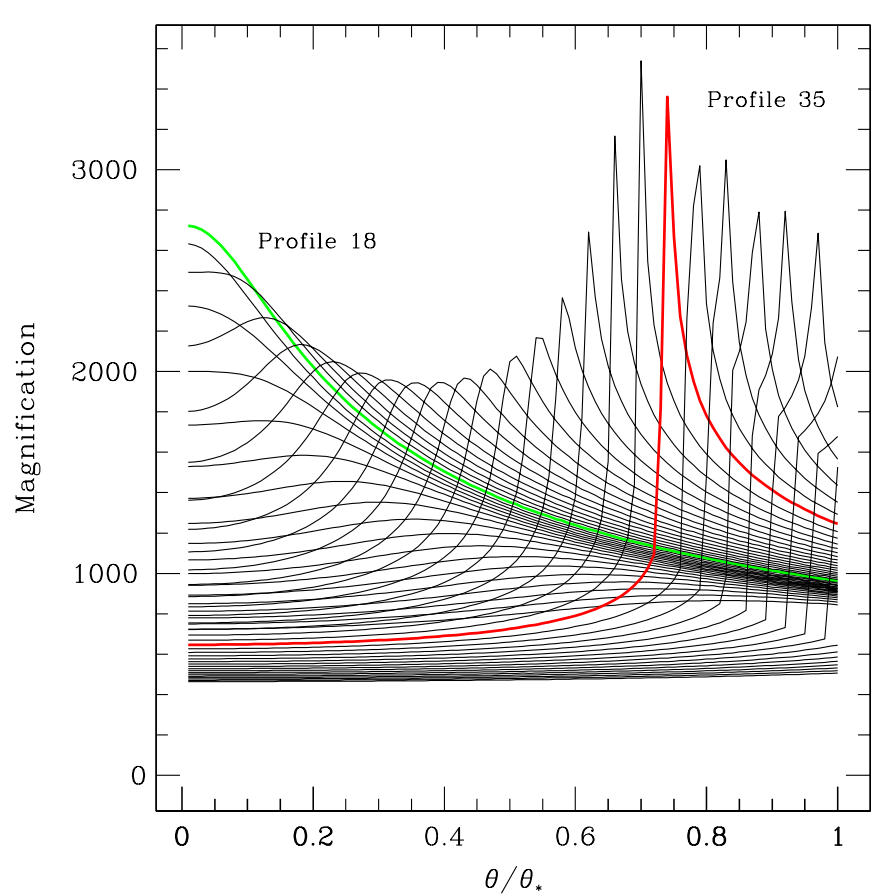

Figure 3. Magnification of the disk of a dwarf as a function of radius at each interval marked in Figure 2. We see in the most extreme cases that parts of the disk are magnified up to $5 \times$ more than other parts. The two profiles that are examined in more detail in the text are for time step 18 (bold line peaking at $\theta=0$ ) and time step 35 (bold line peaking at $\theta=0.75$ )

(A color version of this figure is available in the online journal.)

profiles that occurred during this event. We note that in this case we have ample notice from the light curve that finite source effects are important and we could use that information to derive the magnification profile at the time spectra were taken and make model spectra that include the effects of DLM. However, in this paper, we consider the cases that ignored finite source effects on the spectrum to measure the size of errors that this induces on the parameters, metallicities, and abundance ratios.

\section{SYNTHESIZED SPECTRUM}

We explored the changes to the spectra of dwarfs caused by the magnification profiles given in Section 2. We interpolated a set of model atmospheres from the Kurucz-Castelli grid with new opacity distribution functions (Castelli \& Kurucz 2003). ${ }^{2}$ The models are solar-metallicity and are spaced every $100 \mathrm{~K}$ from $5000 \mathrm{~K}$ to $6200 \mathrm{~K}$. Although the majority of the bulge population is old (Ortolani et al. 1995), we wanted to explore a large temperature range of main-sequence/main-sequence turnoff stars, and therefore we adopted $\log g$ values from a Yale-Yonsei isochrone (Yi et al. 2001) of 4 Gyr. At this age, stars with $T_{\text {eff }}=6000 \mathrm{~K}$ have two possible values for $\log g$, because they are at the turnoff, while dwarfs with $6100 \mathrm{~K}$ and $6200 \mathrm{~K}$ are still present because of the blue hook in the isochrone. The two $6000 \mathrm{~K}$ dwarfs allow us to measure the effect that small changes in $\log g$ have on the resulting spectra. Table 1 lists the temperatures and $\log g$ 's for the dwarfs.

\subsection{Method of Synthesizing Spectra}

We focused our attention on three $200 \AA$ sections of the spectrum, centered on $\mathrm{H} \alpha(6460 \AA-6660 \AA), \mathrm{H} \beta(4757 \AA-$ $4957 \AA$ ) and the Mg I triplet (5067 $\AA-5267 \AA)$. These regions

\footnotetext{
2 Available at http://wwwuser.oat.ts.astro.it/castelli/grids.html
} 
Table 1

Parameters for Model Atmospheres

\begin{tabular}{lccc}
\hline \hline$T_{\text {eff }}(\mathrm{K})$ & $\log g$ & {$[\mathrm{Fe} / \mathrm{H}]$} & $\xi\left(\mathrm{km} \mathrm{s}^{-1}\right)$ \\
\hline 5000 & 4.60 & 0.00 & 1.500 \\
5100 & 4.59 & 0.00 & 1.500 \\
5200 & 4.57 & 0.00 & 1.500 \\
5300 & 4.55 & 0.00 & 1.500 \\
5400 & 4.53 & 0.00 & 1.500 \\
5500 & 4.51 & 0.00 & 1.500 \\
5600 & 4.48 & 0.00 & 1.500 \\
5700 & 4.46 & 0.00 & 1.500 \\
5800 & 4.43 & 0.00 & 1.500 \\
5900 & 4.39 & 0.00 & 1.500 \\
6000 & 4.33 & 0.00 & 1.500 \\
6000 & 4.06 & 0.00 & 1.500 \\
6100 & 4.01 & 0.00 & 1.500 \\
6200 & 3.98 & 0.00 & 1.500 \\
\hline
\end{tabular}

have both strong lines, which are expected to show the largest variations, as well as a number of weaker lines whose equivalent widths would be used in an abundance analysis. The $\mathrm{H} \alpha$ and $\mathrm{H} \beta$ lines are also used as a temperature indicator in hotter dwarfs. For each model atmosphere, we used Turbospectrum (Alvarez \& Plez 1998) to generate intensities $\mathrm{I}(\theta)$ at 100 values of $\theta$. To determine the total flux from the star, we used Equation (1)-(27) from Mihalas (1978) and added up the intensities coming from annuli from the center to the radius $\mathrm{R}$ of the star. For the magnified cases, each annulus was multiplied by its magnification factor before integration. The spectra were smoothed to a FWHM of $0.11 \AA$, or $\mathrm{R} \sim 45,000$, similar to the resolution at which the dwarfs are observed.

\subsection{Comparison of Spectra}

We wished to determine the maximum effect on the spectroscopic analysis, and therefore begin by identifying the cases for which the spectra deviate the most from the unmagnified case. We took the ratio between the magnified and the unmagnified spectra, renormalized the spectra, and then calculated the rms.

For Event A, neither the profiles nor the rms varies much, but the largest deviations are found for the profile calculated for the first time step, indicated by the bold line in Figure 1. For Event $\mathrm{B}$, the rms is highest, as expected, when the limb is magnified by a high factor or when the center region is magnified the most (bold lines in Figure 3). These occur for the 18th and 35th profiles calculated, corresponding to 85 and 170 minutes after Event B began. We will use these three profiles as examples to calculate the size of the effects on the abundances. The indicated case from Event A will be called Profile 1 and the two indicated cases from Event B will be called Profile 18 and 35 .

Figure 4 illustrates the changes to a spectrum by showing the ratio of the unmagnified spectrum to the spectra created using the three different magnification profiles for the $5500 \mathrm{~K}$ dwarf. As expected, the deviations are largest for the two cases where the magnification profile is more extreme and much smaller for Event A, where the differential magnification effects were $\sim 30 \%$, rather than $\sim 500 \%$.

\section{ABUNDANCE ANALYSIS}

We followed the usual steps used to analyze these spectra. Because differential reddening across the face of the bulge leads to uncertainty in the colors and magnitudes of stars in the bulge, analyses of microlensed dwarfs have relied on spectroscopic
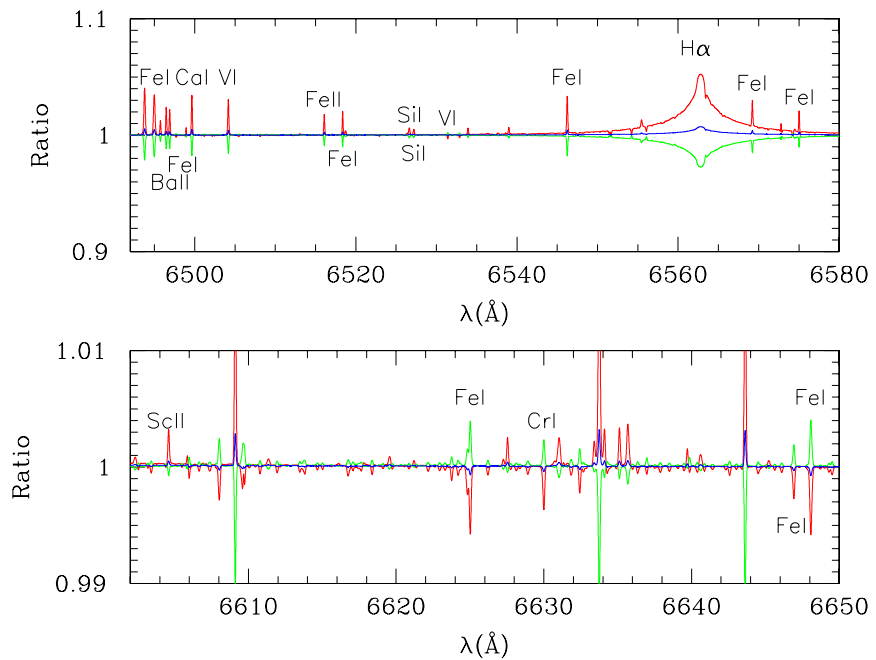

Figure 4. Ratio of the spectra in the magnified cases to those in the unmagnified case for the $5500 \mathrm{~K}$ model. The red line is for Profile 35, the green line is for Profile 18, and the blue line is for Profile 1. Top: part of the H $\alpha$ spectrum, showing the stronger lines, tends to be weaker for Profile 35 than for Profile 18, and in both cases the effects are much stronger than for Profile 1. Bottom: part of the $\mathrm{H} \alpha$ spectrum, focusing on weaker lines, tends to be weaker for Profile 18 than for Profile 35. Again, Profile 1 shows the smallest effects.

(A color version of this figure is available in the online journal.)

methods of deriving atmospheric parameters, rather than color or apparent magnitude-based methods. DLM can impact the derived abundances in the source star by changing the EWs of the lines and by changing the atmospheric parameters derived from those EWs. We first measured EWs in the synthetic spectra for the unmagnified case and Profiles 18, 35, and 1. We ran all sets of EWs through the original model atmosphere and found small changes in the diagnostics used to determine $T_{\text {eff }}$, $\log g$, and $\xi$, in addition to changes in the abundances. We first discuss the magnitude of the changes demanded by the magnified spectra on the atmospheric parameters, considering each one in isolation. While the exact magnitude of the changes depends in detail on which lines are used for any particular study, the results here will give a general indication. However, because deriving the atmospheric parameters usually depends on the other parameters, we next consider the total effect of the accumulated atmospheric parameter changes plus EW changes.

\subsection{Equivalent Width Measurement}

In measuring EWs on the model spectra, we encountered the same concerns about continuum placement and blending as in measuring EWs on observed spectra. Because of our desire to determine differences caused by DLM, we focus on measuring EWs in the same way as on observed spectra and treating the magnified and unmagnified spectra the same. We first used IRAF $^{3}$ to do the continuum division. Then we measured the EWs using SPECTRE (C. Sneden, 2007, private communication) to fit Gaussian profiles to the spectra. Our initial linelist, selected using the solar atlas of Moore et al. (1966), was modified to eliminate lines that deviated by more than 0.1 dex from the mean or that had EW $>150 \mathrm{~m} \AA$. For each temperature, the same lines were measured for each magnification profile. Because the same transition information, such as $g f$-value, was used both

\footnotetext{
3 IRAF is distributed by the National Optical Astronomy Observatory, which is operated by the Association of Universities for Research in Astronomy, Inc. under cooperative agreement with the National Science Foundation.
} 


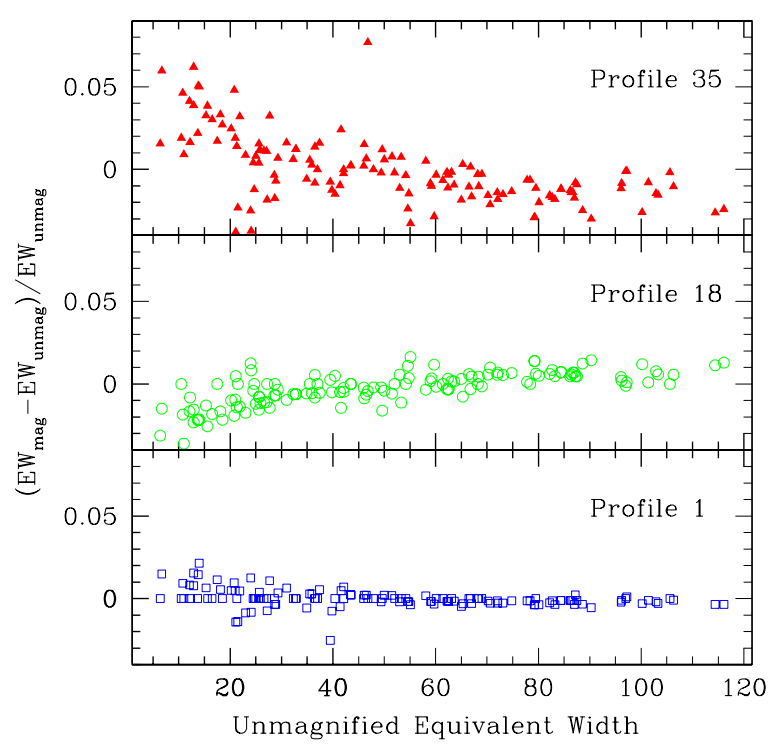

Figure 5. Comparison of the EWs measured in the magnified spectra to those in the unmagnified spectrum for the $5500 \mathrm{~K}$ model. As seen in Figure 4, weaker lines are mostly strengthened and strong lines mostly weakened for Profile 35, while the opposite is true for Profile 18.

to synthesize the spectra and to calculate the abundance, there are no uncertainties introduced in this process by atomic data uncertainties.

In Figure 5, we compare the difference in EWs for the three magnification profiles under study. We find that, as expected, the smaller differences between limb and center for Profile 1 result in smaller changes in the EWs. We then ran the EWs through Turbospectrum, using the original model atmosphere. This analysis reflects the total changes if the analysis relied on non-spectroscopic methods for atmospheric parameter determination, such as color of the unmagnified star for $T_{\text {eff }}$ and position on the CMD for gravity. The changes in abundances are shown in Figure 6, and show the magnitude of $\delta \log \epsilon$ is generally $<0.05$ dex.

\section{2. $T_{\text {eff }}$}

There are two standard ways of determining the temperature of a star from its spectrum, without relying on its color. The first uses the wings of the Balmer lines, while the second requires excitation equilibrium for $\mathrm{Fe}$ I, so that there is no trend in a plot of lower excitation potential to abundance derived for each line.

$\mathrm{H} \alpha$ is well known to show the effects of DLM. Figure 7 compares the $\mathrm{H} \alpha$ profiles of the unmagnified case to the most extreme magnified cases (Profiles 18 and 35) for the $T_{\text {eff }}=$ $5500 \mathrm{~K}$ model. By comparing the magnified cases with the unmagnified profiles of the different temperature models, we determine that the $T_{\text {eff }}$ we would derive would be $75 \mathrm{~K}$ or $100 \mathrm{~K}$ hotter for Profile 18 and would be $75 \mathrm{~K}$ or $100 \mathrm{~K}$ cooler for Profile 35. This is consistent with the magnification profiles for these two cases, because Profile 18 has the center of the star, where the temperatures are hotter at unit optical depth than the limb, magnified more than the limb, while the opposite is true for Profile 35.

Determining the temperature via the wings of the $\mathrm{H} \alpha$ line requires high $\mathrm{S} / \mathrm{N}$ data and becomes increasingly difficult at lower temperatures. Therefore, the more common method, used by all the papers on microlensed dwarfs discussed in the Introduction, is excitation equilibrium. We found that the slope

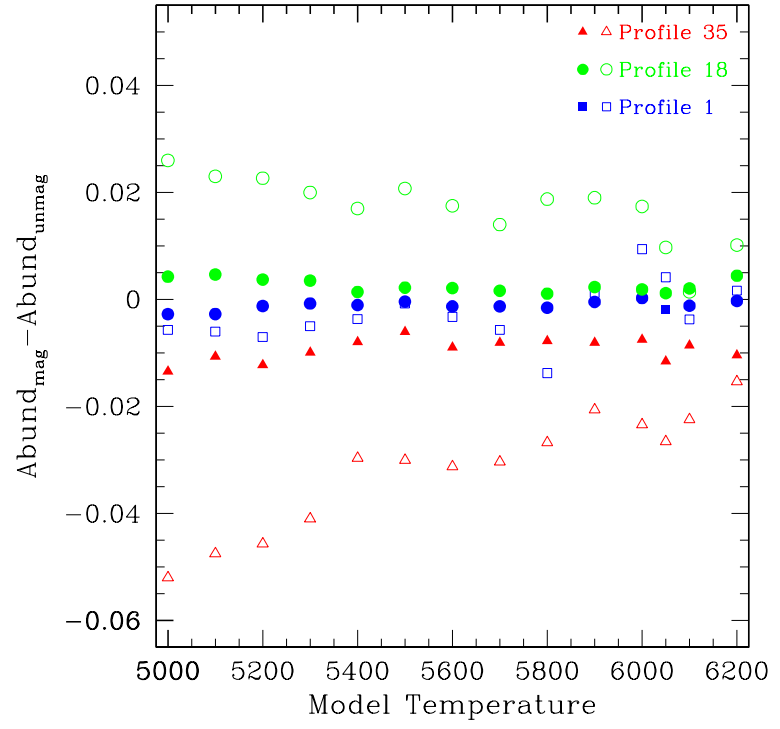

Figure 6. Change in Fe I abundances (filled symbols) and Fe II abundances (open symbols) that result from changes in the EWs alone. Different linelists and continuum division for different temperature models lead to more bumpiness in relation with temperature than otherwise. The lower gravity $6000 \mathrm{~K}$ model points have been offset to $6050 \mathrm{~K}$. Overall, it is clear that the changes in the EWs in themselves do not result in large changes to the abundances. For Profile 18 , for which the center was magnified more than the limb, analyzing the EWs assuming the unmagnified model results in an increase of the derived metallicity, while the reverse is true for Profile 35 . Very small changes are seen for Profile 1, as expected given the small deviations seen in Figure 4.

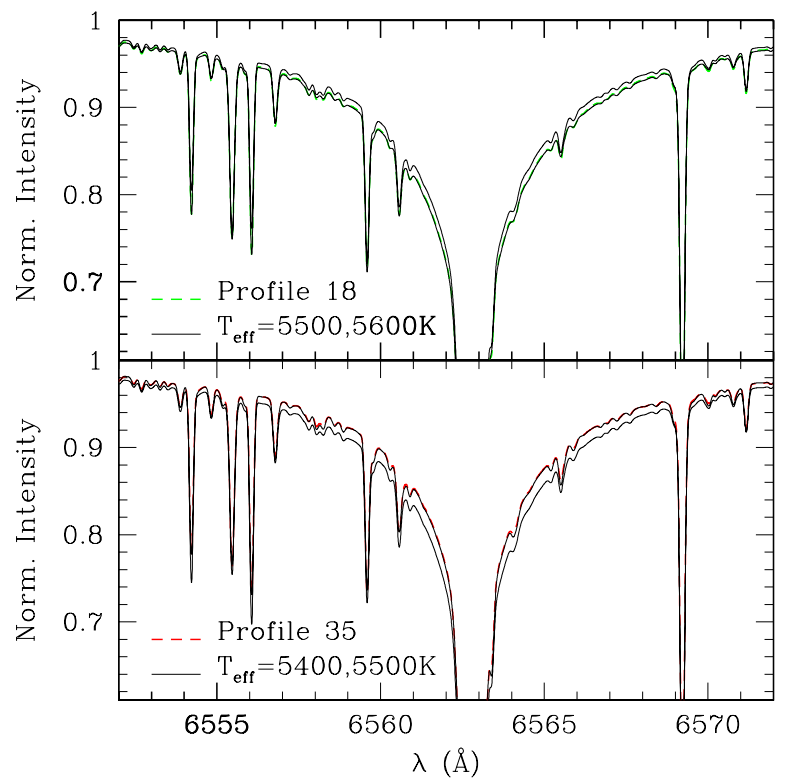

Figure 7. Comparison of unmagnified spectrum at $\mathrm{H} \alpha$ with magnified spectra for Profile 18 (top) and Profile 35 (bottom). The magnified cases are shown for $T_{\text {eff }}=5500 \mathrm{~K}$. In the top panel, the unmagnified cases shown are for $T_{\text {eff }}=$ $5500 \mathrm{~K}$ and $T_{\text {eff }}=5600 \mathrm{~K}$. The slightly broader wings from the Profile 18 spectrum show that we would calculate a higher temperature $(100 \mathrm{~K})$ from this spectrum if we ignored DLM. This agrees with the larger contribution from the center of the star, because its spectrum features stronger Balmer lines. In the bottom panel, the unmagnified cases shown are for $T_{\text {eff }}=5500 \mathrm{~K}$ and $T_{\text {eff }}=5400 \mathrm{~K}$. Here, we would have calculated a lower temperature, which is in agreement with the prominence of the limb, with its weaker Balmer lines, in this particular profile.

of the line, determined by a least-squares fit, in the excitation potential- $\log \epsilon(\mathrm{Fe})$ plane changes if we used the EWs measured from the DLM spectra. Because of errors in continuum division 
and blending, the slope of the line in the unmagnified case, which theoretically should be zero, deviates slightly from that value. We calculated the change in temperature needed to get the slope back to that measured in the unmagnified case. We ran a series of model atmospheres with the temperature decreased by $25 \mathrm{~K}$, found the new slope using the magnified EWs, and used the results to calculate the necessary temperature change.

\subsection{Microturbulent Velocity}

The microturbulent velocity is determined for onedimensional analysis by ensuring that the slope for the derived abundance as a function of reduced $\mathrm{EW}$ for lines of an element is zero. We compared the slopes of the lines in the Fe I versus reduced EW (RW) plot in the magnified case to that in the unmagnified cases using the Fe abundances determined with the original model atmosphere in both cases. As with the $T_{\text {eff }}$, the slope of the line in the unmagnified case is not exactly zero. We run our EWs and original model atmospheres through Turbospectrum, this time with $\xi$ set to $1.4 \mathrm{~km} \mathrm{~s}^{-1}$, and use the change in the slope for $0.1 \mathrm{~km} \mathrm{~s}^{-1}$ to interpolate the change in $\xi$. The magnitude of the change is always $<0.1 \mathrm{~km} \mathrm{~s}^{-1}$.

$$
\text { 4.4. } \log g
$$

For the analysis of the microlensed dwarfs, gravity is determined spectroscopically by demanding that Fe I and Fe II (or other ions) give the same abundance. Obviously, changes in the EWs of these lines as well as changes in $T_{\text {eff }}$ and $\xi$ will result in a change in the derived $\log g$. We find that the changes in $\log g$ necessary to get agreement between Fe I and Fe II, when $\log g$ is in the only parameter changed, are always $<0.1 \mathrm{dex}$.

\subsection{Abundances}

In the previous subsections, we have considered the changes necessary in $T_{\text {eff }}, \xi$, and $\log g$ if we kept the other parameters equal to those of the original model and only modified the one under consideration. This is appropriate when one model atmosphere parameter is chosen independently of the others. However, when deriving a model atmosphere, changes in one parameter will usually propagate and lead to total changes that can be larger or smaller than the partial derivatives suggest.

For our particular linelist, $T_{\text {eff }}, \log g$ and $\xi$ all affect the slope of the excitation potential versus Fe I abundance plot, the slope of the reduced equivalent width versus Fe I abundance plot, and the difference in abundance between Fe I and Fe II lines. Using the partial derivatives we calculated above, we simultaneously solve for the changes in $T_{\text {eff }}, \log g$, and $\xi$ that resulted in the slopes of the two lines and the difference in the two ions being the same as in the unmagnified case. The changes in the atmosphere models from the original parameters in Table 1 are listed in Table 2. The changes are given as magnified parameters versus unmagnified parameters. Finally, we interpolated a model with those parameters and ran the EWs through that model to calculate the final change in the abundances. We note that the metallicity of the model atmosphere will have an effect on the abundances. However, the changes in $\log \epsilon(\mathrm{Fe})$ based on $\mathrm{Fe} I$ lines, which is how the metallicity of the atmosphere is usually set, are small enough that we will ignore this last effect for the purposes of the paper.

Figure 8 shows the resulting changes in the $\log \epsilon$ for the elements considered here for $5000 \mathrm{~K}, 5600 \mathrm{~K}$, and $6200 \mathrm{~K}$. This figure illustrates the small magnitude of the changes expected from DLM in the dwarfs. Also, the changes in abundances are
Table 2

Changes in Parameters for Magnified Model Atmospheres

\begin{tabular}{|c|c|c|c|c|}
\hline Profile & $\Delta T_{\text {eff }}$ & $\Delta \log g$ & $\Delta[\mathrm{Fe} / \mathrm{H}]$ & $\Delta \xi$ \\
\hline \multicolumn{5}{|c|}{ Original $T_{\text {eff }}=5000 \mathrm{~K}$} \\
\hline Profile 1 & -4.0 & -0.006 & -0.005 & 0.002 \\
\hline Profile 18 & 23.5 & 0.024 & 0.008 & 0.031 \\
\hline Profile 35 & -40.3 & -0.039 & -0.018 & -0.059 \\
\hline \multicolumn{5}{|c|}{ Original $T_{\text {eff }}=5100 \mathrm{~K}$} \\
\hline Profile 1 & -4.7 & -0.006 & -0.005 & 0.002 \\
\hline Profile 18 & 23.8 & 0.028 & 0.008 & 0.036 \\
\hline Profile 35 & -31.9 & -0.010 & -0.015 & -0.047 \\
\hline \multicolumn{5}{|c|}{ Original $T_{\text {eff }}=5200 \mathrm{~K}$} \\
\hline Profile 1 & -5.6 & -0.005 & -0.001 & -0.013 \\
\hline Profile 18 & 21.7 & 0.025 & 0.007 & 0.035 \\
\hline Profile 35 & -39.6 & -0.046 & -0.008 & -0.048 \\
\hline \multicolumn{5}{|c|}{ Original $T_{\text {eff }}=5300 \mathrm{~K}$} \\
\hline Profile 1 & -4.7 & -0.005 & -0.001 & -0.013 \\
\hline Profile 18 & 20.6 & 0.025 & 0.008 & 0.032 \\
\hline Profile 35 & -40.2 & -0.049 & -0.018 & -0.065 \\
\hline \multicolumn{5}{|c|}{ Original $T_{\text {eff }}=5400 \mathrm{~K}$} \\
\hline Profile 1 & -4.5 & -0.007 & -0.003 & -0.010 \\
\hline Profile 18 & 20.8 & 0.022 & 0.005 & 0.041 \\
\hline Profile 35 & -36.9 & -0.055 & -0.017 & -0.059 \\
\hline \multicolumn{5}{|c|}{ Original $T_{\text {eff }}=5500 \mathrm{~K}$} \\
\hline Profile 1 & -4.4 & -0.016 & -0.001 & -0.003 \\
\hline Profile 18 & 19.8 & 0.020 & 0.007 & 0.039 \\
\hline Profile 35 & -34.5 & -0.060 & -0.016 & -0.062 \\
\hline \multicolumn{5}{|c|}{ Original $T_{\text {eff }}=5600 \mathrm{~K}$} \\
\hline Profile 1 & -4.2 & -0.007 & -0.004 & -0.002 \\
\hline Profile 18 & 21.2 & 0.026 & 0.008 & 0.041 \\
\hline Profile 35 & -35.7 & -0.052 & -0.020 & -0.063 \\
\hline \multicolumn{5}{|c|}{ Original $T_{\text {eff }}=5700 \mathrm{~K}$} \\
\hline Profile 1 & -5.0 & -0.003 & -0.003 & -0.010 \\
\hline Profile 18 & 20.1 & 0.026 & 0.006 & 0.045 \\
\hline Profile 35 & -38.2 & -0.051 & -0.017 & -0.075 \\
\hline \multicolumn{5}{|c|}{ Original $T_{\text {eff }}=5800 \mathrm{~K}$} \\
\hline Profile 1 & -6.8 & 0.015 & -0.003 & -0.018 \\
\hline Profile 18 & 25.7 & 0.023 & 0.010 & 0.054 \\
\hline Profile 35 & -36.9 & -0.047 & -0.019 & -0.079 \\
\hline \multicolumn{5}{|c|}{ Original $T_{\text {eff }}=5900 \mathrm{~K}$} \\
\hline Profile 1 & -14.4 & -0.043 & -0.007 & -0.013 \\
\hline Profile 18 & 15.9 & 0.002 & 0.005 & 0.044 \\
\hline Profile 35 & -45.6 & -0.094 & -0.023 & -0.073 \\
\hline \multicolumn{5}{|c|}{ Original $T_{\text {eff }}=6000 \mathrm{~K}$, high gravity } \\
\hline Profile 1 & -4.0 & -0.033 & 0.001 & -0.011 \\
\hline Profile 18 & 20.7 & 0.019 & 0.008 & 0.045 \\
\hline Profile 35 & -35.8 & -0.059 & -0.014 & -0.071 \\
\hline \multicolumn{5}{|c|}{ Original $T_{\text {eff }}=6000 \mathrm{~K}$, low gravity } \\
\hline Profile 1 & -6.9 & -0.033 & -0.002 & -0.003 \\
\hline Profile 18 & 18.8 & 0.026 & 0.004 & 0.042 \\
\hline Profile 35 & -39.6 & -0.063 & -0.015 & -0.072 \\
\hline \multicolumn{5}{|c|}{ Original $T_{\text {eff }}=6100 \mathrm{~K}$} \\
\hline Profile 1 & -7.0 & -0.010 & -0.003 & -0.010 \\
\hline Profile 18 & 19.7 & 0.052 & 0.004 & 0.031 \\
\hline Profile 35 & -40.1 & -0.063 & -0.019 & -0.082 \\
\hline \multicolumn{5}{|c|}{ Original $T_{\text {eff }}=6200 \mathrm{~K}$} \\
\hline Profile 1 & -4.9 & -0.020 & 0.000 & -0.014 \\
\hline Profile 18 & 18.1 & 0.033 & 0.007 & 0.034 \\
\hline Profile 35 & -39.2 & -0.096 & -0.019 & -0.073 \\
\hline
\end{tabular}

not always in the same sense. Depending on what part of the source is most highly magnified, the change caused by not taking DLM into account can either be an increase or decrease over 


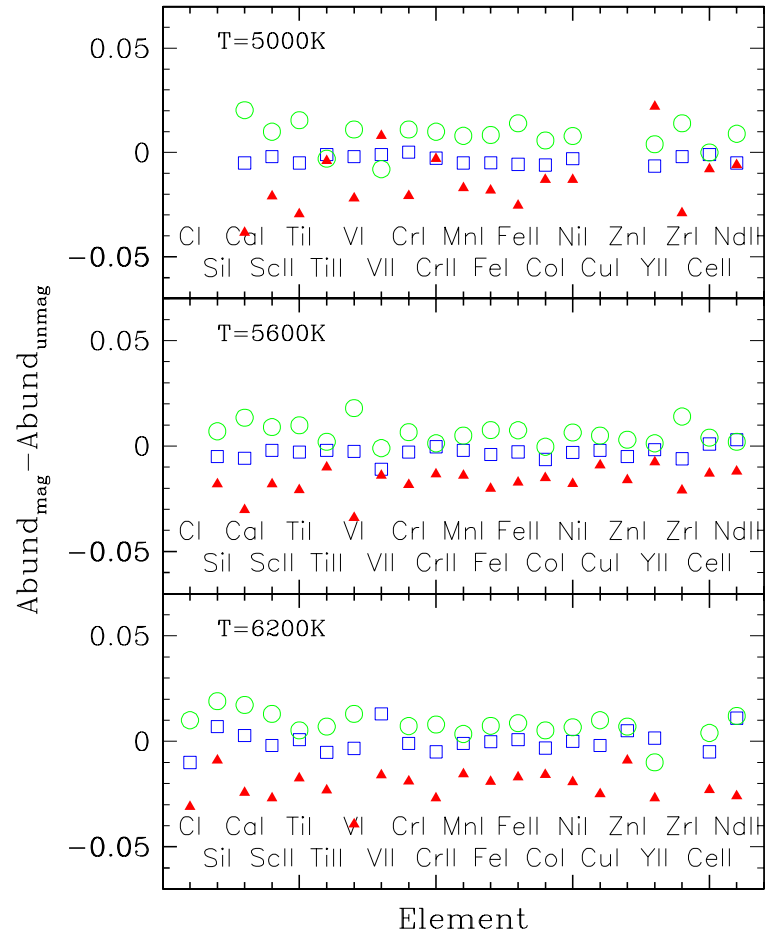

Figure 8. Change in abundance (unmagnified-magnified) from the unmagnified case for three profiles studied in detail in this paper. The results for Profile 1 are shown by the blue squares, for Profile 18 by the green circles, and for Profile 25 by the red triangles. Top: $5000 \mathrm{~K}$ model; middle: $5600 \mathrm{~K}$ case; and bottom: 6200 $\mathrm{K}$ case. Not all elements were measured for each temperature. We see that when DLM is not taken into account while analyzing the spectrum, the abundances can be in error by up to 0.05 dex.

the true abundances in the star. For some ions, such as Fe II, the changes using the new parameters are smaller than those using the new EW with the unmodified atmosphere (seen by comparing Figure 6 and Figure 8). For others, such as Fe I, the changes with the new parameters are larger than seen just using the new EWs.

\section{APPLICATION TO THE STUDY OF MICROLENSED BULGE DWARFS}

As stated in the Introduction, the emphasis on high magnification events means that in many cases there will be DLM when the spectra are taken. Our ability to determine the effect on the spectra from observations falls into four different possibilities: (1) the light curve is indistinguishable from that of a point source and DLM is truly negligible; (2) the light curve is indistinguishable from that of a point source, but DLM occurs; (3) the light curve shows extended source effects, which also means that DLM occurs; and (4) there is no photometric data for crucial parts of the event. Of these possibilities, the first means that the spectrum can be analyzed using standard spectroscopic techniques, while the third can either be analyzed with standard techniques, leading to errors of the order presented here, or analyzed using spectra with the DLM derived from the light curve. These are cases (2) and (4) that are of the most concern for ensuring reliable abundance results. Case (2) is fully addressed by Event A, which illustrates the maximum DLM that is possible without giving rise to noticeable effects, given a well-covered light curve. Case (4), i.e., poor light curve coverage, must be handled on a case-by-case basis. To date, all microlensing events with spectra have had adequate photometric coverage.

\section{CONCLUSION}

Microlensing of bulge dwarfs provides the exciting opportunity to obtain high $\mathrm{S} / \mathrm{N}$ and high-resolution spectra of stars that otherwise would be unfeasible with current telescopes. However, microlensing can produce finite source effects that affect the spectra by differentially magnifying annuli in the star compared to an unmagnified star. We have examined the practical impact of such DLM by synthesizing spectra of dwarfs with magnification profiles similar to events that have been observed and by performing an abundance analysis. We find that, as expected, there are changes to the spectrum, which results in changes in the atmospheric parameters, metallicities, and abundance ratios. Given the small size of the changes, the $\mathrm{S} / \mathrm{N}$ of the observed spectra and the accompanying error in the atmospheric parameters, and the length of the exposures compared to the duration of a specific magnification profile, the resulting effect on the abundances is small compared to other sources of error. We note that the change in abundances can be either positive or negative (see Profile 35 versus Profile 18) depending on the form of the magnification profile. Microlensing events are more commonly in the shape of Event A, with mild magnification causing limb brightening, so there will be a tendency for events to be biased to lower metallicity, but Figure 8 shows that Event A leads to changes of $<0.01$ dex in metallicity. These results, combined with the fact that finite source effects do not affect all of the high-magnification cases for which dwarfs have been observed, demonstrate that this is not the cause of any differences between the observed MDF for giants and dwarfs. Finally, if the light curve shows even more extreme finite source effects than have been modeled in this paper, magnification profiles for that event can be constructed and model spectra can be calculated that appropriately take those profiles into account, so this will not be a limiting problem for the accuracy of abundances derived for microlensed dwarfs.

We thank Carlos Allende Prieto and Judy Cohen for useful comments and discussion. Thomas Masseron provided vital help in the setup and use of Turbospectrum.

\section{REFERENCES}

Abe, F., et al. 2003, A\&A, 411, L493

Afonso, C., et al. 2000, ApJ, 532, 340

Afonso, C., et al. 2001, A\&A, 378, 1014

Albrow, M., et al. 2001, ApJ, 550, L173

Albrow, M. D., et al. 1999, ApJ, 522, 1011

Alcock, C., et al. 1997, ApJ, 491, 436

Alvarez, R., \& Plez, B. 1998, A\&A, 330, 1109

Bensby, T., et al. 2009a, A\&A, 499, 737

Bensby, T., et al. 2009b, ApJ, 699, L174

Bensby, T., et al. 2010, A\&A, accepted (arXiv:0911.5076)

Cassan, A., et al. 2004, A\&A, 419, L1

Cassan, A., et al. 2006, A\&A, 460, 277

Castelli, F., \& Kurucz, R. L. 2003, in IAU Symp. 210, Modeling of Stellar Atmospheres, ed. N. Piskunov, W. W. Weiss, \& D. F. Gray (San Francisco, CA: ASP), 20

Castro, S., Pogge, R. W., Rich, R. M., DePoy, D. L., \& Gould, A. 2001, ApJ, 548, L197

Cohen, J. G., Huang, W., Udalski, A., Gould, A., \& Johnson, J. A. 2008, ApJ, 682,1029

Cohen, J. G., Thompson, I. B., Sumi, T., Bond, I., Gould, A., Johnson, J. A., Huang, W., \& Burley, G. 2009, ApJ, 699, 66

Dong, S., et al. 2006, ApJ, 642, 842

Epstein, C. R., Johnson, J. A., Dong, S., Udalski, A., Gould, A., \& Becker, G. 2010, ApJ, 709, 447

Gaudi, B. S., \& Gould, A. 1999, ApJ, 513, 619

Heyrovský, D., Sasselov, D., \& Loeb, A. 2000, ApJ, 543, 406 
Janczak, J., et al. 2010, ApJ, 711, 731

Johnson, J. A., Gal-Yam, A., Leonard, D. C., Simon, J. D., Udalski, A., \& Gould, A. 2007, ApJ, 655, L33

Johnson, J. A., Gaudi, B. S., Sumi, T., Bond, I. A., \& Gould, A. 2008, ApJ, 685, 508

Kalirai, J. S., Bergeron, P., Hansen, B. M. S., Kelson, D. D., Reitzel, D. B., Rich, R. M., \& Richer, H. B. 2007, ApJ, 671, 748

Kubas, D., et al. 2005, A\&A, 435, 941

Lennon, D. J., Mao, S., Fuhrmann, K., \& Gehren, T. 1996, ApJ, 471, L23

Loeb, A., \& Sasselov, D. 1995, ApJ, 449, L33

Mihalas, D. 1978, Stellar Atmospheres (2nd ed.; San Francisco: Freeman)

Moore, C. E., Minnaert, M. G. J., \& Houtgast, J. 1966, National Bureau of Standards Monograph (Washington, DC: USGPO)
Ortolani, S., Renzini, A., Gilmozzi, R., Marconi, G., Barbuy, B., Bica, E., \& Rich, R. M. 1995, Nature, 377, 701

Ramírez, I., \& Meléndez, J. 2005, ApJ, 626, 465

Thurl, C., Sackett, P. D., \& Hauschildt, P. H. 2006, A\&A, 455, 315

Valls-Gabaud, D. 1995, in Large Scale Structure in the Universe, ed. J. P. Muecket, S. Gottloeber, \& V. Mueller (Singapore: World Scientific), 326

Valls-Gabaud, D. 1998, MNRAS, 294, 747

Witt, H. J. 1995, ApJ, 449, 42

Yi, S., Demarque, P., Kim, Y.-C., Lee, Y.-W., Ree, C. H., Lejeune, T., \& Barnes, S. 2001, ApJS, 136, 417

Zoccali, M., Hill, V., Lecureur, A., Barbuy, B., Renzini, A., Minniti, D., Gómez, A., \& Ortolani, S. 2008, A\&A, 486, 177

Zoccali, M., et al. 2003, A\&A, 399, 931 